\title{
Discriminação racial e saúde: ações dos profissionais de saúde na assistência à mulher em processo de abortamento provocado
}

\author{
Racial discrimination and health: health professionals' actions \\ in providing care women in the induced abortion process
}

Aldo Pacheco Ferreira (https://orcid.org/0000-0002-7122-5042) ${ }^{1}$

Gisela Cordeiro Pereira Cardoso (https://orcid.org/0000-0002-4014-0951) ${ }^{2}$

Cíntia da Silva Telles Nichele (https://orcid.org/0000-0001-6685-1017) ${ }^{3}$

Vania Reis Girianelli (https://orcid.org/0000-0002-8690-9893) ${ }^{1}$

Angélica Baptista Silva (https://orcid.org/0000-0003-0292-5106) ${ }^{1}$

Marluce Rodrigues Godinho (https://orcid.org/0000-0002-6570-4645) ${ }^{4}$
${ }^{1}$ Departamento de Direitos Humanos, Saúde e Diversidade Cultural, Escola Nacional de Saúde Pública Sergio Arouca (ENSP), Fundação Oswaldo Cruz (Fiocruz). Av. Brasil 4036, Manguinhos. 21040-361 Rio de Janeiro RJ Brasil. aldopachecoferreira@ gmail.com

${ }^{2}$ ENSP, Fiocruz. Rio de Janeiro RJ Brasil.

${ }^{3}$ Programa de Pós-

Graduação em Saúde

Pública, ENSP, Fiocruz. Rio

de Janeiro RJ Brasil.

${ }^{4}$ Departamento de

Enfermagem Básica,

Faculdade de Enfermagem,

Universidade Federal de Juiz

de Fora. Juiz de Fora MG

Brasil.

\begin{abstract}
This paper aims to evaluate the racial inequalities in the care provided by health professionals concerning induced abortion. This systematic review study used the Preferred Reporting Items for Systematic Reviews and Meta-Analyzes (PRISMA) model, based on the following bases: Brazilian Virtual Health Library (BVS), Scientific Electronic Library Online (SciELO), National Library of Medicine, and National Institutes of Health (PubMed), Science Direct, Capes periodicals portal, with the descriptors: "racism OR social discrimination AND abortion, induced AND health personnel $O R$ comprehensive health care $O R$ delivery of health care OR human rights", selected via the DeCS and Medical Subject Heading (MeSH). Eighteen papers published between 2005 and 2020 in national and international literature were analyzed following the inclusion and exclusion criteria. Most studies found a significant relationship between racial discrimination and institutional violence, including access and quality of care for patients undergoing an induced abortion. Racial discrimination is a significant risk factor for adverse care outcomes.
\end{abstract}

Key words Abortion, Health professional, Racial discrimination, Emergency medical services, $\mathrm{Hu}$ man Rights
Resumo Este artigo tem como objetivo avaliar as desigualdades raciais na assistência de profissionais de saúde ao abortamento provocado. Estudo de revisão sistemática, seguindo o modelo Preferred Reporting Items for Systematic Reviews and Meta-Analyses (PRISMA), a partir das seguintes bases: Biblioteca Virtual em Saúde, Scientific Eletronic Library Online (SciELO), National Library of Medicine and National Institutes of Health (PubMed), Science Direct, Portal de Periódicos Capes, com os descritores: "racism OR social discrimination AND abortion, induced AND health personnel OR comprehensive health care OR delivery of health care OR human rights", escolhidos mediante consulta ao DeCS e Medical Subject Heading (MeSH). Seguindo os critérios de inclusão e exclusão, analisaram-se 18 artigos, publicados entre os anos de 2005 a 2020 na literatura nacional e internacional. A maioria dos estudos encontrou uma relação significativa entre discriminação racial e violência institucional, incluindo o acesso e qualidade da assistência de pacientes em abortamento provocado. A discriminação racial é um fator de risco significativo para resultados adversos no atendimento.

Palavras-chave Aborto, Profissional de saúde, Discriminação racial, Serviços de saúde de emergência, Direitos Humanos 


\section{Introdução}

O racismo é uma causa negligenciada, mas relevante, das disparidades de saúde em sociedades multiétnicas ${ }^{1}$. Diferentes tipos de racismo e outras expressões de discriminação devem ser reconhecidos, analisados criticamente e ativamente revertidos. Empiricamente distinguimos e reconhecemos as omissões e violações dos direitos humanos e, em seguida, analisamos as fontes do racismo em estreita relação com uma visão interseccional sobre as formas de discriminação baseadas no gênero, classe e raça ${ }^{2}$. A maioria das sociedades é racista e esse fenômeno se vincula entre racismo e vulnerabilidade resultando em iniquidades na saúde. Hoje em dia, o racismo é identificado como uma preocupação de saúde relevante, mas negligenciada e muitas vezes ignorada $^{3-6}$.

Os direitos humanos não são um dado, mas um constructo, uma elaboração humana, em constante processo de construção e reconstrução ${ }^{7}$. Conforme aponta Piovesan ${ }^{8}$ : Enquanto reivindicação moral, os direitos humanos nascem quando devem e podem nascer. Tendo em consideração a perspectiva temporal desses direitos, constata-se que o sentido dos direitos humanos significa uma multiplicidade de acepções; dentre as quais, destaca-se o entendimento contemporâneo caracterizado pela universalidade e pela indivisibilidade desses direitos ${ }^{9}$, fundamentado na Declaração Universal ${ }^{10}$, e subsequentemente confirmado na Declaração de Direitos Humanos de Viena ${ }^{11}$. Santos ${ }^{12}$ ainda acrescenta: "temos o direito a ser iguais quando nossa diferença nos inferioriza; e temos o direito a ser diferentes quando nossa igualdade nos descaracteriza. Daí a necessidade de uma igualdade que reconheça as diferenças e de uma diferença que não produza, alimente ou reproduza as desigualdades".

Vários autores discutem sobre marginalização e cobertura de serviços de saúde, ao identificarem uma carência significativa em relação ao acesso à cobertura médica ${ }^{13,14}$, configurando-se de tal forma como um racismo institucionalizado, proporcionado pelo acesso discriminatório a instalações, bens, serviços ${ }^{15}$. Consequentemente, o racismo institucionalizado é evidente ainda nas sociedades, porém mimetizada nas diversas práticas sociais ${ }^{16}$. O racismo mediado pessoalmente está relacionado ao preconceito e à discriminação com base na raça, o qual pode ser intencional ou não; porém, se manifesta devido ao desrespeito, desconfiança, desvalorização, acusação e desumanização ${ }^{17}$.
Com efeito, o direito humano à saúde, também conhecido como o direito ao mais alto padrão de saúde possível, compreende componentes internacionais juridicamente vinculativos. Um dos componentes mais importantes para o direito à saúde é o Pacto Internacional sobre Direitos Econômicos, Sociais e Culturais (PIDESC); especialmente o Comentário Geral no 14 do PIDESC ${ }^{18}$. $\mathrm{O}$ direito à saúde com base no Comentário Geral $n^{\circ} 14$ engloba elementos essenciais avaliados pela estrutura de quatro indicadores cruciais: disponibilidade, acessibilidade, aceitabilidade e qualidade (Quadro 1). Disponibilidade refere-se à existência e quantidade de instalações, bens e serviços de saúde. A acessibilidade se concentra no acesso físico e econômico aos bens e serviços das unidades de saúde. Além disso, a acessibilidade tem quatro dimensões: não discriminação, física, econômica e acesso à informação. A aceitabilidade está relacionada à sensibilidade das instalações, bens e serviços de saúde à cultura e à ética médica. Em termos de instalações de saúde de qualidade, os bens e serviços devem ser científica e medicamente adequados e de qualidade satisfatória ${ }^{19}$.

Pesquisas anteriores em países multiétnicos do primeiro mundo como os Estados Unidos ${ }^{20}$, Reino Unido $^{21}$, Austráliaa ${ }^{22}$ e Nova Zelândia ${ }^{23}$, mas também em países multiétnicos em desenvolvimento, como o Brasil ${ }^{24}$, México ${ }^{25}$ e comparativamente Brasil, México, Colômbia e Peru ${ }^{26}$ enfatizaram o racismo como a causa das disparidades de saúde persistentes. Por exemplo, uma pesquisa sediada nos Estados Unidos sobre disparidades de saúde por raça forneceu evidências de desigualdades significativas entre a população afro-americana e a população branca do país. Nos Estados Unidos, os afro-americanos têm taxas de mortalidade mais altas do que suas contrapartes brancas para a maioria das principais causas de morte ${ }^{27}$. No entanto, as disparidades de saúde não são determinadas biológica ou culturalmente; elas são explicadas por uma estrutura complexa de fatores sociais, econômicos e políticos ${ }^{28}$. Portanto, disparidades na saúde relacionadas ao racismo são um argumento crucial para a importância dos determinantes sociais da saúde ${ }^{29,30}$. Em relação aos direitos humanos, o não racismo e de outras discriminações são posições a serem consideradas ${ }^{31}$; notadamente, a acessibilidade às instalações, bens e serviços de saúde sem discriminação é fundamental ${ }^{32}$.

Tendo em vista a necessidade de sistematização do conhecimento acumulado nos últimos anos, a presente proposta visa avaliar a discriminação racial na assistência à mulheres com quadro de abortamento por profissionais de saúde. 
Quadro 1. Estrutura referente ao Pacto Internacional sobre Direitos Econômicos, Sociais e Culturais (PIDESC).

\begin{tabular}{|l|l|}
\hline \multicolumn{1}{|c|}{ Dados } & \multicolumn{1}{c|}{ Definição } \\
\hline Disponibilidade & $\begin{array}{l}\text { Deve ter uma quantidade suficiente de instalações, bens e serviços públicos de saúde em } \\
\text { funcionamento e programas }\end{array}$ \\
\hline Acessibilidade & $\begin{array}{l}\text { Instalações, bens e serviços de saúde devem ser acessíveis (ou seja, acesso físico, financeiro e } \\
\text { de informações) a todos dentro da jurisdição do Estado, sem discriminação }\end{array}$ \\
\hline Aceitabilidade & $\begin{array}{l}\text { A distância social e cultural entre os sistemas de saúde e seus usuários determina a } \\
\text { aceitabilidade. Todas as instalações, bens e serviços de saúde devem respeitar a ética médica e } \\
\text { ser culturalmente apropriados e sensíveis ao sexo e à idade. Elas também devem ser elaboradas } \\
\text { para respeitar confidencialmente e melhorar o estado de saúde dos indivíduos envolvidos }\end{array}$ \\
\hline Qualidade & $\begin{array}{l}\text { As instalações, bens e serviços de saúde devem ser cientificamente e medicamente aprovadas } \\
\text { e de qualidade satisfatória }\end{array}$ \\
\hline
\end{tabular}

Fonte: PIDESC ${ }^{18}$.

\section{Métodos}

Trata-se de uma revisão sistemática que tem como base as recomendações do Preferred Reporting Items for Systematic Reviews and Meta-Analyses $(\text { PRISMA })^{33}$. Foi realizada busca eletrônica de artigos nas bases de dados da Biblioteca Virtual em Saúde (BDENF - Enfermagem, BINACIS, IBECS, LILACS, MEDLINE), Scientific Eletronic Library Online (SciELO), National Library of Medicine and National Institutes of Health (PubMed), Science Direct, Portal de Periódicos Capes (JSTOR Archival Journals, OneFile (GALE), Science Citation Index Expanded (Web of Science), Scopus (Elsevier), Social Sciences Citation Index (Web of Science), Sociological Abstracts (ProQuest), Taylor \& Francis (online - Journals), com os descritores "racism OR social discrimination AND abortion, induced AND health personnel OR comprehensive health care OR delivery of health care OR human rights", escolhidos mediante consulta aos Descritores em Ciência da Saúde (DeCS) e Medical Subject Heading (MeSH).

Foram incluídos estudos publicados independentemente do ano de publicação, nos idiomas inglês, espanhol e português, e que avaliassem a discriminação racial na assistência por profissionais de saúde em situação de aborto provocado. Foram excluídos os artigos de revisão bibliográfica, artigos no formato de teses, dissertações, monografias, editoriais e relatos de caso; e aqueles que não atendessem a $80 \%$ dos itens requeridos pelas escalas de avaliação da qualidade metodológica utilizadas neste estudo. Para cada estudo incluído foram extraídos os seguintes dados: autores, ano de publicação e de realização do trabalho, desenho do estudo, local, população estudada, desfecho avaliado, limitações metodológicas e principais resultados.
A qualidade dos estudos observacionais foi avaliada pela escala Strengthening the reporting of observational studies in epidemiology (STROBE) $)^{34}$. Esta escala propõe uma lista de 22 itens que devem estar presentes no corpo dos artigos para que sejam considerados de qualidade. Em sua versão traduzida e validada no Brasil em $2008^{35}$, foram estabelecidas categorias de qualidade dos artigos, sendo que os artigos que preenchem $80 \%$ ou mais dos itens da lista são considerados categoria "A"34,35. O Standards for Reporting Qualitative Research (SRQR) ${ }^{36}$, foi utilizado para avaliação dos estudos qualitativos. Os estudos com metodologia quanti-qualitativa foram analisados por ambos os instrumentos de qualidade. Foram incluídos neste artigo os estudos que alcançaram pontuação igual ou superior a $80 \%$ em pelo menos uma das duas escalas. A avaliação da elegibilidade temática dos artigos foi realizada por dois revisores independentes e a avaliação metodológica por apenas um dos revisores.

\section{Resultados}

Foram identificados 3.826 artigos, sendo que 1.135 foram excluídos por estarem repetidos nas bases de dados. Após análise dos títulos e resumos, 2.477 artigos foram excluídos por não atenderem aos critérios de elegibilidade da pesquisa. Os 214 restantes foram lidos e analisados na íntegra. Destes, 182 foram excluídos por não atenderem aos critérios de inclusão. Os 32 remanescentes foram analisados conforme as escalas STROBE e SRQR, sendo 14 excluídos por não terem atenderem a pontuação mínima de $80 \%$ dos itens, restando 18 artigos. A Figura 1 apresenta o processo de seleção dos artigos.

No Quadro 2 são apresentadas as características gerais dos estudos selecionados com a distri- 
buição dos trabalhos segundo o ano de publicação, a região geográfica onde foram realizados, o caminho metodológico, amostra e objetivo. Cerca de $78 \%$ dos artigos foram publicados a partir de 2011.

O levantamento dos artigos sem restrição temporal proporcionou a observação da evolução da série histórica. O primeiro artigo encontrado é de 2005, depois em sequência de 2007, 2008 e 2009, e a temática reaparece somente em 2011, observando-se a partir daí publicações em todos os anos subsequentes até o presente de 2020.

$\mathrm{Na}$ análise da assistência por profissionais de saúde, a percepção dos sujeitos sobre o aborto provocado e das mulheres enquanto potenciais mães se somam aos sentidos atribuídos ao hospital-maternidade para influenciar negativamente a qualidade do atendimento, levando à objetificação das mulheres em abortamento ${ }^{45}$. De modo geral, o conjunto de profissionais ao serem questionados acerca dos possíveis motivos que levam as mulheres a engravidar e abortar, sem distinção de categoria ocupacional, descreve as usuárias que induziram o aborto, incluindo uma ou mais das seguintes características: negras, precárias condições financeiras, baixa escolaridade, instabilidade do laço conjugal, abandono do parceiro, falta de apoio familiar, irresponsáveis e de sexualidade desenfreada ${ }^{38,39,44,47,48,51,52}$.

Em alguns casos observou-se uma diferença nos profissionais de acordo com o tipo de aborto, provocado ou não; ou seja, para aquelas mulheres que sofreram um aborto espontâneo, cria-se uma imagem de fragilidade e de reconhecimento do seu sofrimento, mas classificam àquelas que o induziram como agressivas, arredias, aliviadas e indiferentes. Estas últimas são desqualificadas ${ }^{24,29}$, sendo atribuído um estado de anormalidade psíquica ao praticarem um aborto.

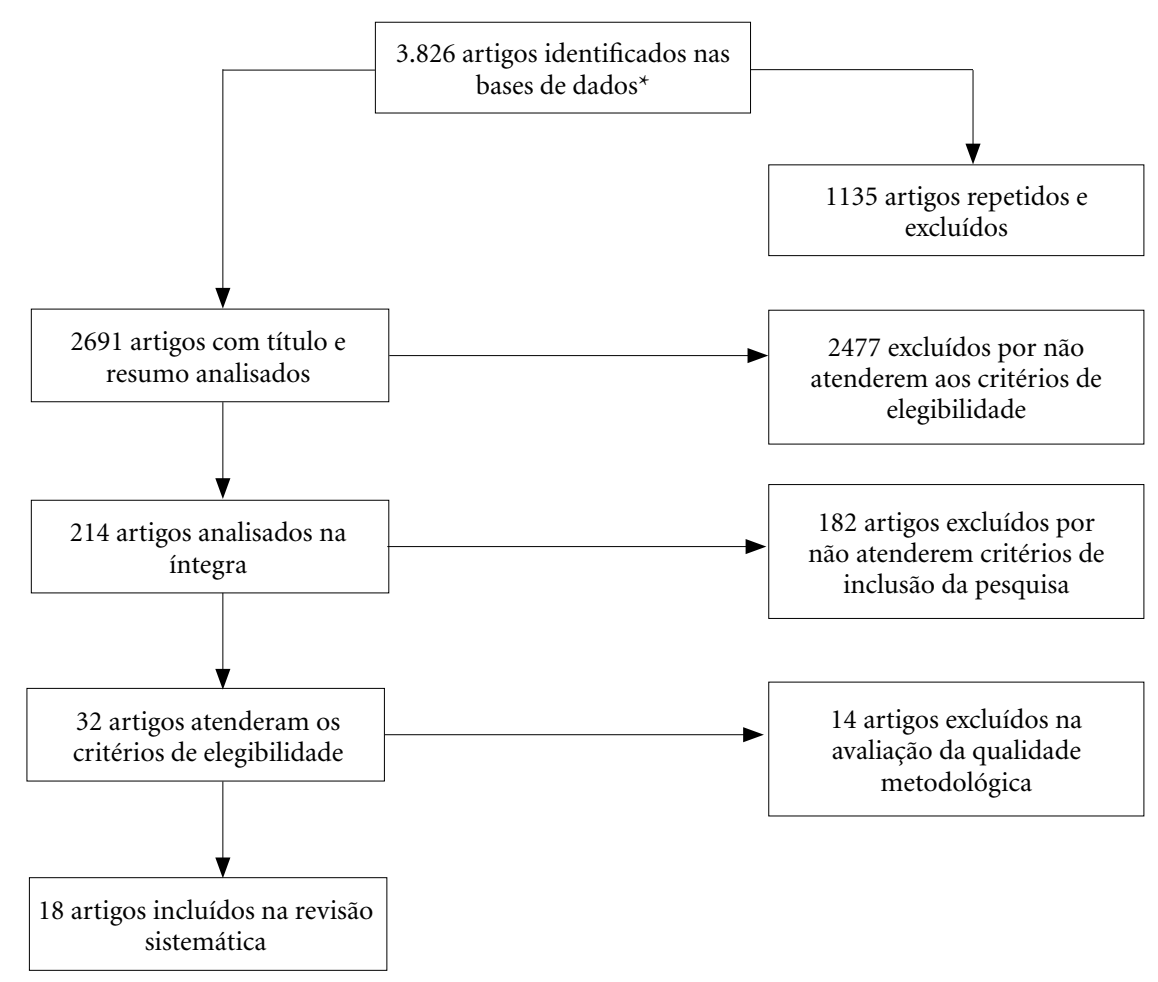

Figura 1. Diagrama de fluxo da seleção de artigos para a revisão sistemática, 2020.

*Bases de dados consultadas: Biblioteca Virtual em Saúde (BDENF - Enfermagem, BINACIS, IBECS, LILACS, MEDLINE), Scientific Eletronic Library Online (SciELO), National Library of Medicine and National Institutes of Health (PubMed), Science Direct, Portal de Periódicos Capes (JSTOR Archival Journals, OneFile (GALE), Science Citation Index Expanded (Web of Science), Scopus (Elsevier), Social Sciences Citation Index (Web of Science), Sociological Abstracts (ProQuest), Taylor \& Francis Online - Journals). 


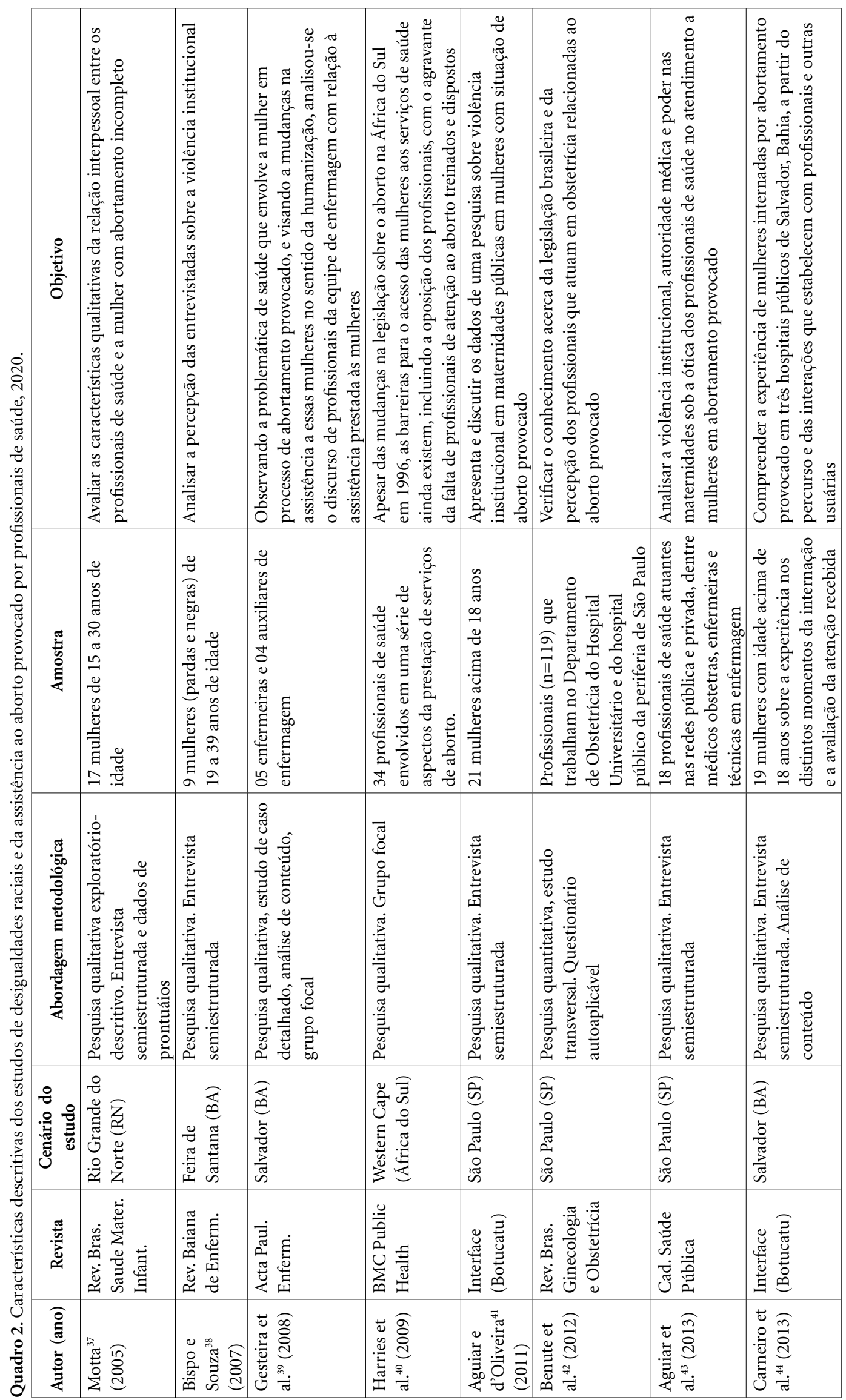


离

\begin{tabular}{|c|c|c|c|c|c|c|c|c|}
\hline & $\frac{\stackrel{8}{*}}{\frac{0}{0}}$ & 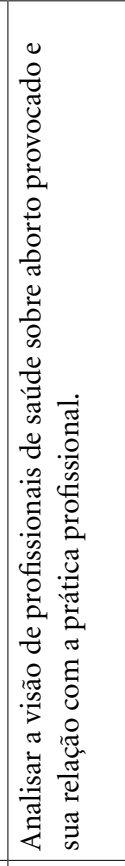 & 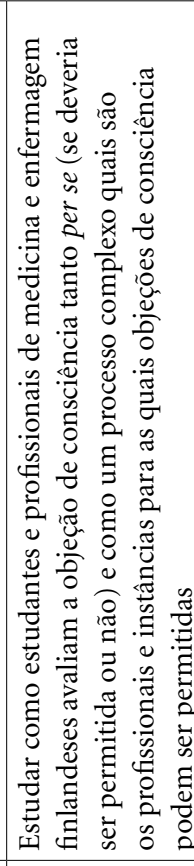 & 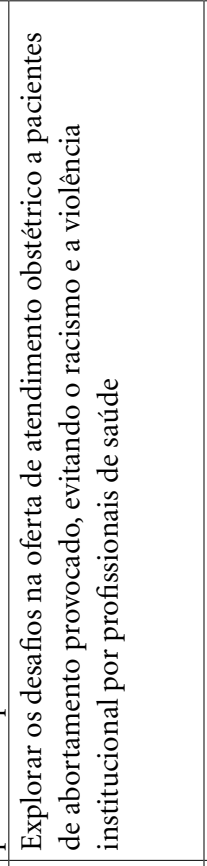 & 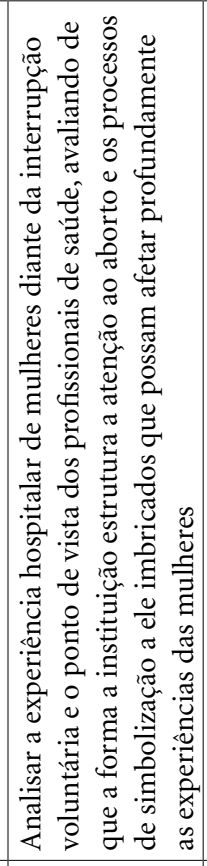 & 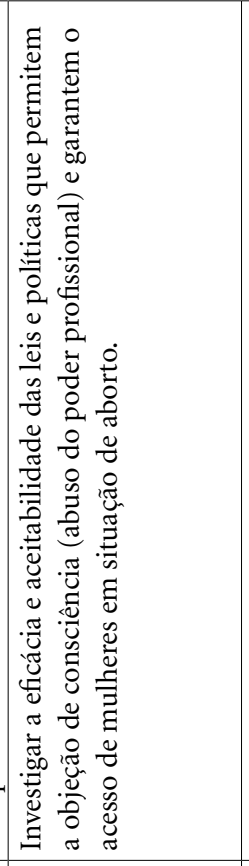 & 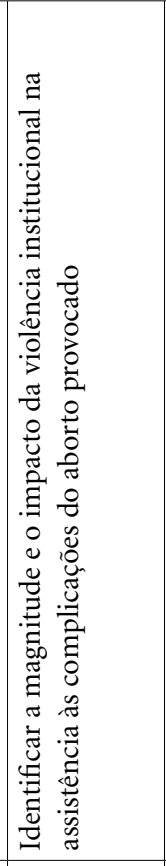 & 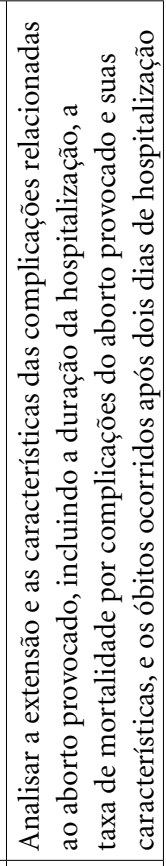 \\
\hline & $\begin{array}{l}\mathbb{5} \\
\stackrel{5}{0} \\
\stackrel{3}{4}\end{array}$ & 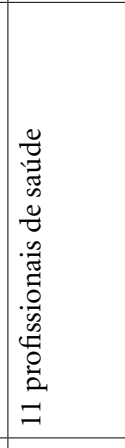 & 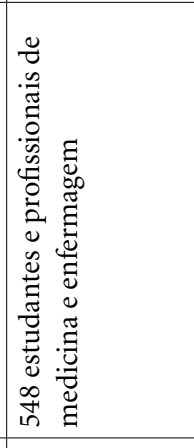 & 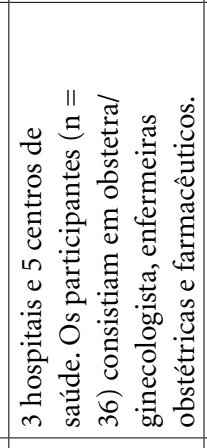 & 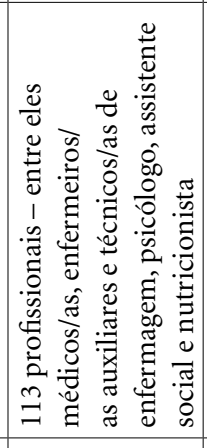 & 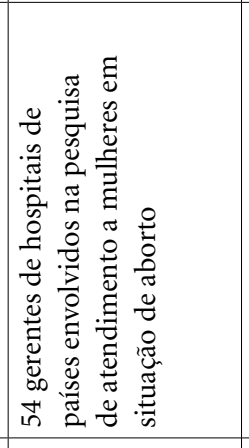 & 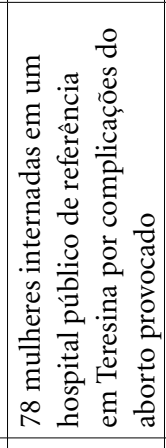 & 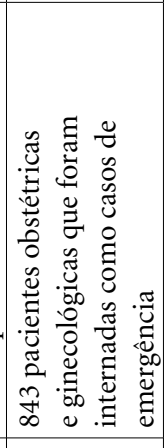 \\
\hline & 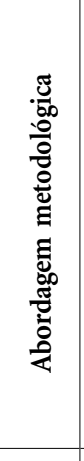 & 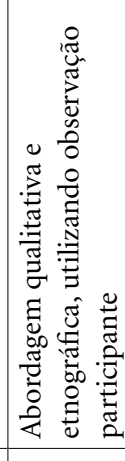 & 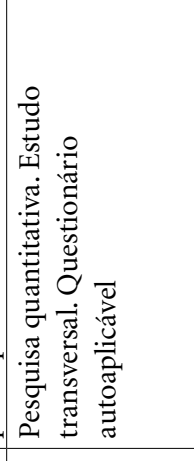 & 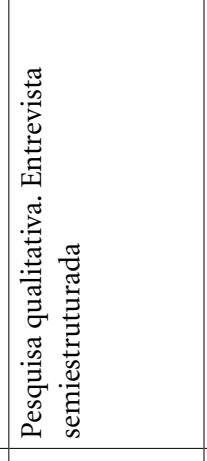 & 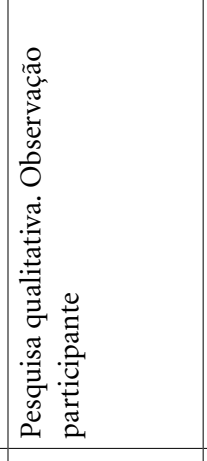 & 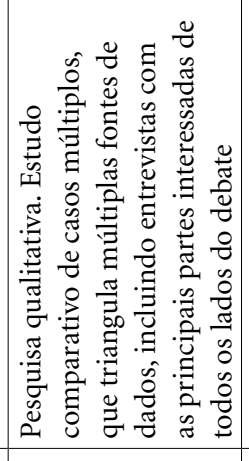 & 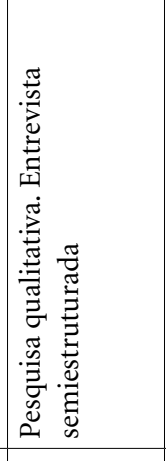 & 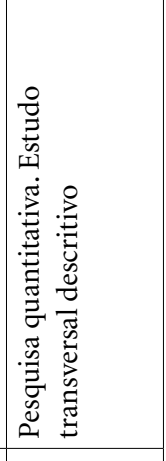 \\
\hline & 赵 & 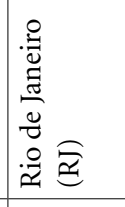 & 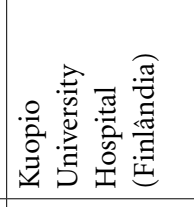 & 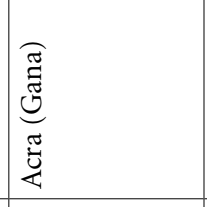 & 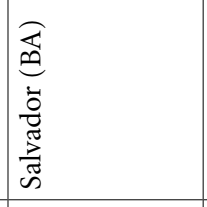 & 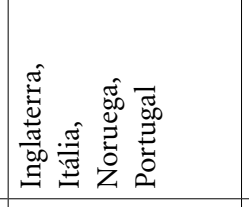 & 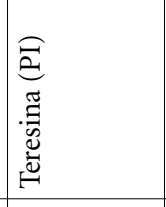 & 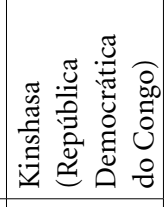 \\
\hline & : & 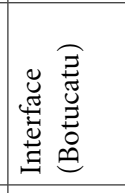 & 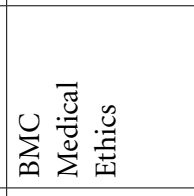 & 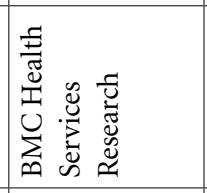 & 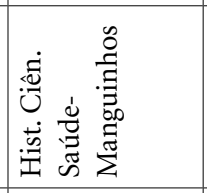 & 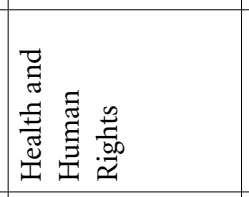 & 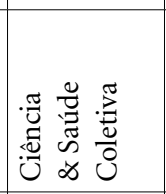 & $\begin{array}{l}\text { y } \\
z \\
0 \\
0 \\
0 \\
\vdots \\
\end{array}$ \\
\hline & 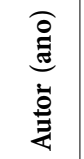 & 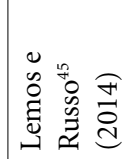 & 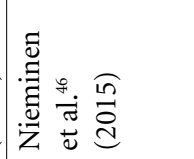 & 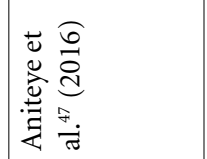 & 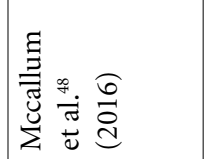 & 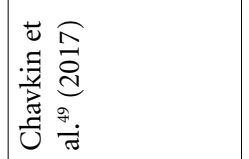 & 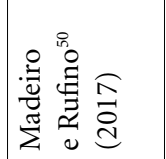 & 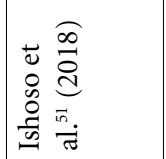 \\
\hline
\end{tabular}




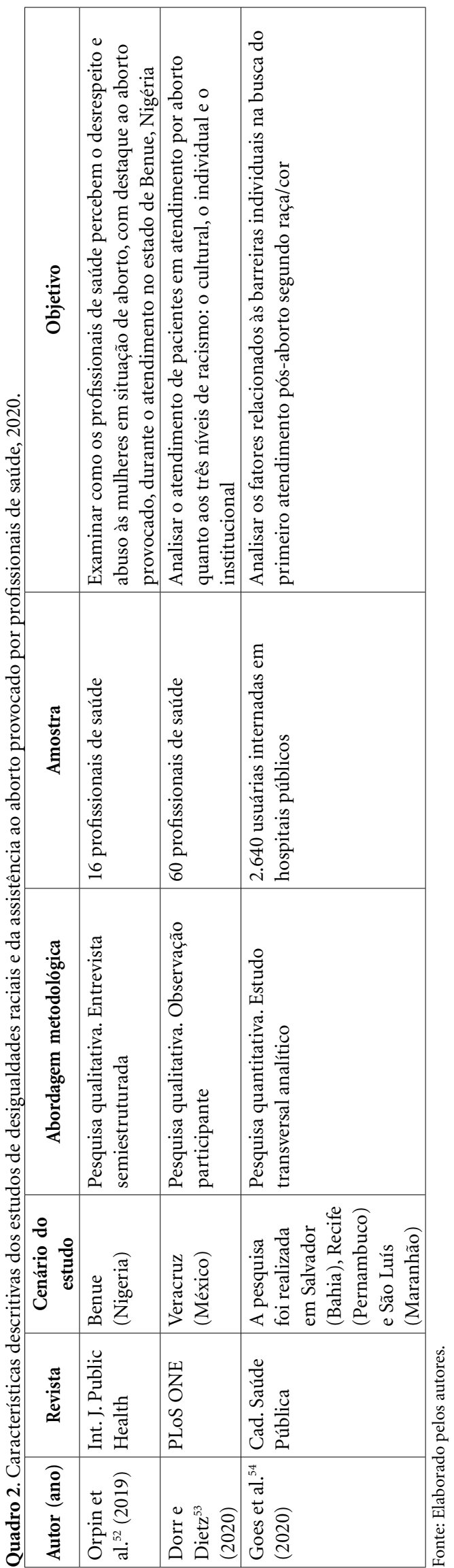

Os resultados expressam indícios de que as instituições de saúde e os profissionais desses hospitais abarcam em suas práticas a violência institucional ${ }^{38,40,41,43,49,53}$, sobretudo com práticas desumanizantes de atenção ${ }^{51,52}$ e violência simbólica $^{52}$. Ademais, a não priorização do atendimento às mulheres com abortamento é evidente na pouca atenção dada a estas usuárias, e a existência de discriminação racial. São identificados nos artigos as precárias condições das unidades de saúde, deficiências da infraestrutura dos serviços, com superlotação das maternidades ${ }^{44,47,51-54}$.

$\mathrm{Na}$ análise dos serviços obstétricos foi possível perceber duas áreas nas quais a literatura associa a presença e reprodução da violência institucional: de um lado os conceitos e valores dos próprios cuidadores frente ao aborto ${ }^{43,53} \mathrm{e}$ ao cuidado prestado nas maternidades ${ }^{49,51} \mathrm{e}$, do outro, as normas institucionais e o processo de traba$\mathrm{lho}^{37,39,42}$. Combinados, estes fatores evidenciam atitudes e práticas que remetem à violência institucional. Na maioria dos casos de abortamento provocado os estudos apontaram a violência física, por meio da não oferta de medicamentos, sejam analgésicos ou anestésicos, para aliviar a dor ${ }^{52,53}$. No caso da violência verbal aparece o uso de questionamentos da moral das mulheres sendo, também, verbalmente estigmatizadas ${ }^{46,47}$, com despersonalização e constrangimento ${ }^{41}$.

No Brasil, Gesteira et al. ${ }^{39}$ assinalam a influência do contexto de ilegalidade do aborto e dos valores morais da sociedade nas percepções dos profissionais sobre a má qualidade da assistência. Entretanto, neste estudo a discussão de seus resultados aborda muito rapidamente os elementos do contexto social mais amplo, sem uma análise aprofundada sobre o fenômeno da violência institucional.

Alguns estudos discutem os condicionamentos socioculturais que influenciam os profissionais e suas práticas de cuidado ${ }^{51-53}$. Os autores constatam atitudes de pré-julgamento dos profissionais e suas dificuldades em se despirem de suas convicções pessoais na atenção às mulheres. No entanto, as atitudes e os conflitos éticos são analisados desconsiderando-se o contexto institucional imediato da maternidade e os processos políticos historicamente constituídos que definem as possibilidades de atuação das instituições e dos indivíduos que nela atuam.

Harries et al. $^{40}$ reconhecem o contexto que envolve as práticas institucionais, nas quais estão presentes o baixo investimento na qualificação dos trabalhadores, a fragmentação e verticalização do processo de trabalho, com implicações na 
relação entre próprios trabalhadores e entre estes e os usuários; e assim, é admitida também a insuficiente capacitação de profissionais e gestores para lidarem com as questões sociais e subjetivas que interferem nas práticas de atenção à saúde.

Em geral, as percepções dos profissionais de saúde frente as mulheres em situação de abortamento provocado foram marcadas por valores de cunho negativo, que influenciaram os sentidos e significados atribuídos a essa prática e, consequentemente sobre as mulheres atendidas, responsabilizando-as pela gravidez e sua interrupção, com atitudes discriminadoras sendo naturalizadas $^{39}$.

\section{Discussão}

A partir da exposição dos principais achados, considera-se que o estudo atendeu aos objetivos propostos, trazendo resultados e descobertas importantes sobre as desigualdades raciais pela análise da assistência ao aborto provocado por profissionais de saúde. Estudos relataram nas pacientes abuso verbal e físico, desumanização no atendimento, falta de privacidade e confidencialidade, maus-tratos, e atitudes negativas e hostis da equipe como uma barreira à utilização de serviços qualificados foram relatados ${ }^{39,47,51,52}$. Semelhante aos achados desta revisão, fatores como equipe, infraestrutura, equipamentos e suprimentos inadequados e falta de supervisão dos profissionais de saúde também foram descritos como fatores importantes que contribuem para

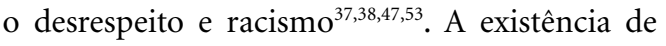
desrespeito e racismo foi essencialmente definida como atitude hostil, pobre ou negativa da equi$\mathrm{pe}^{48,53}$.

O racismo ocorre em vários níveis, incluindo institucional, interpessoal e internalizado. O racismo institucionalizado resulta em acesso diferenciado aos recursos, serviços e oportunidades da sociedade por raça. Com relação aos recursos, o racismo institucionalizado inclui disparidades no acesso e na qualidade da educação, moradia segura, emprego, recursos de saúde e condições ambientais, onde as mesmas combinadas com a falta de oportunidades de influenciar políticas, limitam o poder das minorias raciais e étnicas de tomar e fazer cumprir decisões. O racismo interpessoal se refere ao preconceito e à discriminação resultando em suposições diferenciadas sobre as capacidades e os motivos dos outros de acordo com sua raça. A discriminação inclui o tratamento diferenciado de outras pessoas com base em sua raça e é mais comumente considerada racismo ${ }^{15,16}$. Finalmente, o racismo internalizado resulta na aceitação de atributos, competências e valor negativos pelos membros de grupos marginalizados. O racismo internalizado limita a capacidade de alguém atingir seu potencial máximo ${ }^{55}$.

Foram identificados somente três estudos de abordagem quantitativa, o que impacta por inexistir informações amplas sobre as opiniões, atitudes e conhecimentos dos profissionais de saúde com relação ao aborto, em especial ao provocado, que foi o foco da busca. Talvez isso se deva a uma eventual falta de respaldo para a realização de pesquisas de maior abrangência com esses profissionais, uma vez que as pesquisas quantitativas, geralmente aplicadas em amostras bastante numerosas, demandam de questionários padronizados, validados e confiáveis que possam ser utilizados para essa finalidade, além de significativos montantes de recursos financeiros e apoio institucional. Outro resultado que chamou atenção foi a inexistência de dados sobre profissionais de saúde mental.

Nossos resultados de revisão evidenciaram pesquisas onde mulheres de minorias raciais e étnicas experimentam maior exposição ao longo da vida a estressores crônicos, o que pode aumentar seu risco de resultados ruins na gravi$\mathrm{dez}^{56}$. O acúmulo de estresse ao longo da vida de uma mulher, conhecido como carga alostática, está associado a piores resultados de saúde ${ }^{57}$. A discriminação racial é um desses estressores crônicos que pode ser um fator de risco para resultados adversos no nascimento. As definições de racismo são variadas, mas todas incluem o conceito de tratamento desigual decorrente da cor da pele ou de outras características individuais ${ }^{55}$.

Por fim, outro dado que, talvez, necessite ser pesquisado com maior profundidade, e, se possível, com mais amplitude, é a percepção de atitudes racistas no atendimento clínico em situação de aborto. A literatura aponta pesquisas que sugerem uma ampla aceitação dos casos de aborto permitidos por lei ${ }^{58-61}$, e, além disso, nota-se que as situações de malformação fetal grave também contam com a aprovação da grande maioria dos profissionais de saúde ${ }^{62}$.

\section{Conclusão}

Apesar de suas limitações, esta revisão tem uma série de implicações importantes para um campo de pesquisa que tem recebido pouca atenção. Abortos provocados são comuns, e as mulheres 
que os praticam sofrem vários tipos e graus de estigma. O racismo e outras ideologias relacionadas, como sexismo e classismo, são preocupações de saúde relevantes, embora a aplicação na prática pareça insuficiente com base nas evidências de desrespeito e abuso. Nossos dados demonstraram, como o racismo institucionalizado se manifesta na falta de recursos humanos qualificados, escassez de produtos de saúde funcionais em termos de quantidade e qualidade e discriminação dependente de renda dentro das instalações médicas. Destacamos também exemplos de internalização por vítimas de racismo e reprodução de discriminação por profissionais de saúde.

O fortalecimento da capacidade do sistema de saúde através do emprego de um número ade- quado de profissionais de saúde, especialmente em áreas onde há uma necessidade não atendida, junto com a melhoria nas instalações de infraestrutura, também reduzirá o desrespeito e as práticas de abuso interpretadas como causadas por problemas como superlotação nas instalações e falta de profissionais de saúde.

As percepções sociais subjacentes apontam para a necessidade de campanhas de conscientização e intervenções educacionais em níveis sociopolíticos e comunitários mais amplos, incluindo a educação de mulheres e homens sobre os direitos a cuidados respeitosos. Por fim, fica denotado que o racismo viola o direito à saúde em todas as dimensões.

\section{Colaboradores}

AP Ferreira trabalhou na concepção, pesquisa, metodologia e redação final. MR Godinho e CST Nichele trabalharam na pesquisa, metodologia e redação final. VR Girianelli trabalhou na metodologia e redação final. AB Silva e GCP Cardoso trabalharam na concepção e redação final.

\section{Agradecimentos}

À Coordenação de Aperfeiçoamento de Pessoal de Nível Superior (CAPES), pela bolsa de Doutorado de CST Nichele. Ao Conselho Nacional de Desenvolvimento Científico e Tecnológico (CNPq), pela Bolsa de Produtividade em Pesquisa de AP Ferreira. 


\section{Referências}

1. Souza J. Raça ou classe? Sobre a desigualdade brasileira. Lua Nova 2005; 65:43-69.

2. Espósito YB. Subjetivação necropolítica e a materialidade do pós-estruturalismo. Agenda Polit 2020; 8(1):313-336.

3. Williams DR, Mohammed SA. Discrimination and racial disparities in health: evidence and needed research. J Behav Med 2009; 32(1):20-47.

4. Johnstone M-J, Kanitsaki O. The neglect of racism as an ethical issue in health care. J Immigr Minor Health 2010; 12(4):489-495.

5. Horton R. Offline: Racism - the pathology we choose to ignore. Lancet 2017; 390(10089): 14 .

6. Bhopal RS. Intertwining migration, ethnicity, racism, and health. Lancet 2017; 390(10098):932.

7. Arendt H. As origens do totalitarismo. São Paulo: Cia. das Letras; 1989.

8. Piovesan F. Direitos sociais, econômicos e culturais e direitos civis e políticos. Sur Rev Int Direitos Hum 2004; 1(1):20-47.

9. Dworkin R. Levando os direitos a sério. São Paulo: Martins Fontes; 2007.

10. Assembleia Geral das Nações Unidas. Declaração Universal dos Direitos Humanos [internet]. [acessado 29 set 2020]. Disponível em: https://www.unidosparaosdireitoshumanos.com.pt/what-are-human-rights/universal-declaration-of-human-rights/articles-01-10.html.

11. Biblioteca Virtual de Direitos Humanos da USP. Declaração e Programa de Ação de Viena [Internet]. [acessado 29 set 2020]. Disponível em: http://www. direitoshumanos.usp.br/index.php/Sistema-Global.Declara\%C3\%A7\%C3\%B5es-e-Tratados-Internacionais-de-Prote $\% \mathrm{C} 3 \% \mathrm{~A} 7 \% \mathrm{C} 3 \% \mathrm{~A} 3 \mathrm{o} /$ declaracao-e -programa-de-acao-de-viena.html.

12. Santos BS. Reconhecer para libertar: Os caminhos do cosmopolitismo multicultural. Rio de Janeiro: Civilização Brasileira; 2003.

13. Travassos C, Viacava F, Fernandes C, Almeida CM. Desigualdades geográficas e sociais na utilização de serviços de saúde no Brasil. Cien Saude Colet 2000; 5(1):133-149.

14. Viacava F, Porto SM, Carvalho CC, Bellido JG. Desigualdades regionais e sociais em saúde segundo inquéritos domiciliares (Brasil, 1998-2013). Cien Saude Colet 2019; 24(7):2745-2760.

15. Silvério VR. Ação afirmativa e o combate ao racismo institucional no Brasil. Cad Pesqu 2002; 117:219-246.

16. Silva MAB. Racismo Institucional: pontos para reflexão. Laplage Rev 2017; 3(1):127-136.

17. Modesto JG, Minelli AC, Fernandes MP, Rodrigues M, Bufolo R, Bitencourt R, Pilati R. Racismo e Políticas Afirmativas: Evidências do Modelo da Discriminação Justificada. Psicol Teoria Pesqu 2017; 33:e3353.

18. Pacto Internacional sobre Direitos Econômicos, Sociais e Culturais (PIDESC). General comment no. 14. The right to the highest attainable standard of health (article 12 of the International Covenant on Economic, Social and Cultural Rights). Genebra: ONU; 2000.

19. Minayo MCS, Hartz ZMA, Buss PM. Qualidade de vida e saúde: um debate necessário. Cien Saude Colet 2000; 5(1):7-18.
20. Barnes LL, De Leon CFM, Wilson RS, Bienias JL, Bennett DA, Evans DA. Racial differences in perceived discrimination in a community population of older blacks and whites. J Aging Health 2004; 16(3):315-337.

21. Karlsen S, Nazroo JY. Relation between racial discrimination, social class, and health among ethnic minority groups. Am J Public Health 2002; 92(4):624-631.

22. Larson A, Gillies M, Howard PJ, Coffin J. It's enough to make you sick: the impact of racism on the health of Aboriginal Australians. Aust N Z J Public Health 2007; 31(4):322-329.

23. Harris R, Tobias M, Jeffreys M, Waldegrave K, Karlsen $S$, Nazroo J. Racism and health: The relationship between experience of racial discrimination and health in New Zealand. Soc Sci Med 2006; 63(6):1428-1441.

24. Lemos A, Russo JA. Healthcare professionals and abortion: what is said and not said in a professional healthcare capacitation. Interface (Botucatu) 2014; 18(49):301-312.

25. Bautista JER, López AO. Muerte materna en mujeres indígenas de México y racismo de Estado. Dispositivos biopolíticos en salud. Rev Salud Problema 2017; 11(21):28-53.

26. Perreira KM, Telles EE. The color of health: skin color, ethnoracial classification, and discrimination in the health of Latin Americans. Soc Sci Med 2014; 116:241250.

27. Kung H-C, Hoyert DL, Xu J, Murphy SL. Deaths: final data for 2005. Natl Vital Stat Rep 2008; 56(10):1-120.

28. Smedley BD, Stith AY, Nelson AR. Unequal treatment. Confronting racial and ethnic disparities in health care. Washington, D.C.: National Academies Press; 2003.

29. Ramaswamy M, Kelly PJ. Institutional racism as a critical social determinant of health. Public Health Nurs 2015; 32(4):285-286.

30. Paradies Y, Ben J, Denson N, Elias A, Priest N, Pieterse A, Gupta A, Kelaher M, Gee G. Racism as a determinant of health: A systematic review and meta-analysis. PLoS One 2015; 10(9):e0138511.

31. Bazotti KDV, Stumm EMF, Kirchner RM. Ser cuidada por profissionais da saúde: Percepções e sentimentos de mulheres que sofreram abortamento. Texto Contexto Enferm 2009; 18(1):147-154.

32. Hunt P, Backman G. Health systems and the right to the highest attainable standard of health. Health Hum Rights 2008; 10(1):81-92.

33. Liberati A, Altman DG, Tetzlaff J, Mulrow C, Gotzsche PC, Ioannidis JP, Clarke M, Devereaux PJ, Kleijnen J, Moher D. The PRISMA statement for reporting systematic reviews and meta-analyses of studies that evaluate health care interventions: explanation and elaboration. PLoS Med 2009; 6(7):e1000100.

34. Vandenbroucke JP, Von Elm E, Altman DG, Gotzsche PC, Mulrow CD, Pocock SJ, Poole C, Schlesselman JJ, Egger M, Strobe Initiative. Strengthening the reporting of observational studies in epidemiology (STROBE): explanation and elaboration. PLoS Med 2007; 4(10):e297.

35. Malta M, Cardoso LO, Bastos FI, Magnanini MM, Silva CM. STROBE initiative: guidelines on reporting observational studies. Rev Saude Publica 2010; 44(3):559-565. 
36. O’Brien BC, Harris IB, Beckman TJ, Reed DA, Cook DA. Standards for reporting qualitative research: a synthesis of recommendations. Academic Med 2014; 89(9):1245-1251.

37. Motta IS. A relação interpessoal entre profissionais de saúde e a mulher em abortamento incompleto: "o olhar da mulher". Rev Br Saude Materno Infantil 2005; 5(2):219-228.

38. Bispo CDB, Souza VLC. Violência institucional sofrida por mulheres internadas em processo de abortamento. Rev Baiana Enferm 2007; 21(1):19-30.

39. Gesteira SMA, Diniz NMF, Oliveira EM. Assistência à mulher em processo de abortamento provocado: discurso de profissionais de enfermagem. Acta Paulista Enferm 2008; 21(3):449-453.

40. Harries J, Stinson K, Orner P. Health care providers' attitudes towards termination of pregnancy: A qualitative study in South Africa. BMC Public Health 2009; 9:296.

41. Aguiar JM, d'Oliveira AFPL. Violência institucional em maternidades públicas sob a ótica das usuárias. Interface (Botucatu) 2011; 15(36):79-92.

42. Benute GRG, Nonnenmacher D, Nomura RMY, Lucia MCS, Zugaib M. Influência da percepção dos profissionais quanto ao aborto provocado na atenção à saúde da mulher. Rev Br Ginecol Obstetr 2012; 34(2):6973.

43. Aguiar JM, d'Oliveira AFPL, Schraiber LB. Violência institucional, autoridade médica e poder nas maternidades sob a ótica dos profissionais de saúde. Cad Saude Publica 2013; 29(11):2287-2296.

44. Carneiro MF, Iriart JAB, Menezes GMS. "Largada sozinha, mas tudo bem": paradoxos da experiência de mulheres na hospitalização por abortamento provocado em Salvador, Bahia, Brasil. Interface (Botucatu) 2013; 17(45):405-418.

45. Lemos A, Russo JA. Profissionais de saúde e o aborto: o dito e o não dito em uma capacitação profissional em saúde. Interface (Botucatu) 2014; 18(49):301-312.

46. Nieminen P, Lappalainen S, Ristimäki P, Myllykangas $\mathrm{M}$, Mustonen AM. Opinions on conscientious objection to induced abortion among Finnish medical and nursing students and professionals. BMC Med Ethics 2015; 16:17.

47. Aniteye P, O'Brien B, Mayhew SH. Stigmatized by association: challenges for abortion service providers in Ghana. BMC Health Serv Res 2016; 16:486.

48. Mccallum C, Menezes G, Reis AP. O dilema de uma prática: experiências de aborto em uma maternidade pública de Salvador, Bahia. Hist Cien Saude-Manguinhos 2016; 23(1):56.

49. Chavkin W, Swerdlow L, Fifield J. Regulation of conscientious objection to abortion: An international comparative multiple-case study. Health Hum Rights 2017; 19(1):55-68.

50. Madeiro AP, Rufino AC. Maus-tratos e discriminação na assistência ao aborto provocado: a percepção das mulheres em Teresina, Piauí, Brasil. Cien Saude Colet 2017; 22(8):2771-2780.
51. Ishoso DK, Tshefu AK, Coppieters Y. Analysis of induced abortion-related complications in women admitted to referral health facilities in Kinshasa, Democratic Republic of the Congo. PLoS One 2018; 13(8):e0203186.

52. Orpin J, Puthussery S, Burden B. Healthcare providers' perspectives of disrespect and abuse in maternity care facilities in Nigeria: a qualitative study. Int $J$ Public Health 2019; 64:1291-1299.

53. Dorr NM, Dietz G. Racism against Totonaco women in Veracruz: Intercultural competences for health professionals are necessary. PLoS One 2020; 15(1):e0227149.

54. Goes EF, Menezes GMS, Almeida MCC, Araújo TVB, Alves SV, Alves MTSSB, Aquino EML. Vulnerabilidade racial e barreiras individuais de mulheres em busca do primeiro atendimento pós-aborto. Cad Saude Publica 2020; 36(Supl. 1):e00189618.

55. Jones CP. Levels of racism: a theoretic framework and a gardener's tale. Am J Public Health 2000; 90:12121215.

56. Latendresse G. The interaction between chronic stress and pregnancy: preterm birth from a biobehavioral perspective. J Midwifery Womens Health 2009; 54:817.

57. Sousa MBC, Silva HPA, Galvão-Coelho NL. Resposta ao estresse: I. omeostase e teoria da alostase. Estud Psicol 2015; 20(1):2-11.

58. Menezes M, Aquino EML. Pesquisa sobre o aborto no Brasil: avanços e desafios para o campo da saúde coletiva. Cad Saude Publica 2009; 25(Supl. 2):S193-S204.

59. Diniz D, Penalva J, Faúndes A, Rosas C. A magnitude do aborto por anencefalia: um estudo com médicos. Cien Saude Colet 2009; 14(Supl. 1):1619-1624.

60. Diniz D, Madeiro A, Rosas C. Conscientious objection, barriers, and abortion in the case of rape: a study among physicians in Brazil. Reprod Health Matters 2014; 22(43):141-148.

61. Drezett J, Pedroso D, Gebrim LH, Matias ML, Macedo $\mathrm{Jr} \mathrm{H}$, Abreu LC. Motivos para interromper legalmente a gravidez decorrente de estupro e efeitos do abortamento nos relacionamentos cotidianos das mulheres. Reprod Climatério 2011; 26:85-91.

62. Patrício SS, Gregório VRP, Pereira SM, Costa R. Malformação fetal com possibilidade de interrupção legal: dilemas maternos. Rev Br Enferm 2019; 72(Supl. 3):125-131.

Artigo apresentado em 20/11/2020

Aprovado em 17/05/2021

Versão final apresentada em 19/05/2021

Editores-chefes: Romeu Gomes, Antônio Augusto Moura da Silva 
